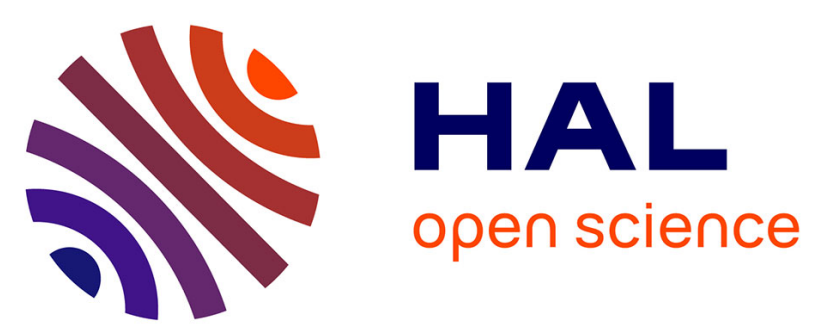

\title{
Actuator and Friction Dynamics Formulation in Control of PKMs: From Design to Real-Time Experiments
}

\author{
Hussein Saied, Ahmed Chemori, Maher El Rafei, Clovis Francis, François
}

Pierrot

\section{- To cite this version:}

Hussein Saied, Ahmed Chemori, Maher El Rafei, Clovis Francis, François Pierrot. Actuator and Friction Dynamics Formulation in Control of PKMs: From Design to Real-Time Experiments. IROS: Intelligent RObots and Systems, Oct 2018, Madrid, Spain. pp.5634-5639, 10.1109/IROS.2018.8594329 . lirmm-01887156

\section{HAL Id: lirmm-01887156 \\ https://hal-lirmm.ccsd.cnrs.fr/lirmm-01887156}

Submitted on 3 Oct 2018

HAL is a multi-disciplinary open access archive for the deposit and dissemination of scientific research documents, whether they are published or not. The documents may come from teaching and research institutions in France or abroad, or from public or private research centers.
L'archive ouverte pluridisciplinaire HAL, est destinée au dépôt et à la diffusion de documents scientifiques de niveau recherche, publiés ou non, émanant des établissements d'enseignement et de recherche français ou étrangers, des laboratoires publics ou privés. 


\title{
Actuator and Friction Dynamics Formulation in Control of PKMs: From Design to Real-Time Experiments
}

\author{
Hussein Saied ${ }^{1,2}$, Ahmed Chemori ${ }^{1}$, Maher El Rafei ${ }^{2}$, Clovis Francis $^{2}$ and Francois Pierrot ${ }^{1}$
}

\begin{abstract}
This paper deals with a new dynamic formulation of parallel manipulators incorporating the actuator and friction dynamics to be utilized in control. A model-based controller, PD with computed feedforward, is implemented for a parallel robot taking into consideration the formulated dynamics. The motivation behind this contribution is to enhance the control performance by compensating the unfavourable nonlinearities abundant extensively in PKMs. Those nonlinearities may increase considerably when operating at high-speed motions. The proposed feedforward part relies on the reference trajectories instead of the measured ones improving the control performance and the computational efforts. To validate our contribution, real-time experiments are conducted on a four degree-of-freedom parallel robot named VELOCE in different operating conditions.
\end{abstract}

\section{INTRODUCTION}

During the last decades, Parallel Kinematic Manipulators (PKMs) have occupied the first rank in most of the industrial tasks. According to [1], any structure made up of a fixed base and traveling plate linked together with at least two independent kinematic chains is considered a PKM.

The advantages of PKMs are: High precision, high payload-to-weight ratio, high acceleration capabilities and a good dynamic response. On the other hand, PKMs suffer from some limitations like the small workspace and the limited rotational motion, the complexity in the forward kinematic solutions, and the considerable number of singularities [2]. From the wide range of applications served by PKMs, we can mention the following: food packaging [1], flight simulators [3], machining tasks [4] and medical applications [5]. In order to get use of their above mentioned advantages in these applications, several control techniques have been proposed in the literature [6], [7], [8], [9]. Control of PKMs, known with their high nonlinearity, time-varying parameters and uncertainties, is considered as a real challenging task. Non-model-based control schemes have shown acceptable tracking performances, but still weak against the parameters variation and uncertainties which are abundant in parallel robots. Model-based control schemes deal with the nonlinearity of parallel robots and may attain better performances than non-model-based control schemes thanks to the dynamic model incorporated in the closed-loop control algorithm [10].

\footnotetext{
${ }^{1}$ Hussein Saied, Ahmed Chemori and Francois Pierrot are with Laboratoire d'Informatique, de Robotique, et de Microlectronique de Montpellier (LIRMM), Univ. Montpellier - CNRS, 161 rue Ada, 34095 Montpellier, France [saied, chemori,pierrot] @lirmm.fr

${ }^{2}$ Hussein Saied, Maher El Rafei and Clovis Francis are with the Scientific Research Center in Engineering (CRSI), Lebanese Univ., Faculty of Engineering, Hadath, Beirut, Lebanon [maher.elrafei,cfrancis] @ul.edu.lb
}

Due to the good performance improvement resulting from incorporating the dynamic model of a PKM in the closedloop controller, the need for an accurate and comprehensive model arises. Most of the existing control techniques ignore the effect of the actuator and friction dynamics for simplicity purposes, or take into consideration only one factor of them considering different assumptions. In [11], [12], simulations were performed with linked-serial manipulator example to verify the validity of some back-stepping control techniques incorporating both manipulator and actuator dynamics. Realtime experiments in [13] are performed on the MIT Serial Link Direct Drive Arm using computed torque control methods incorporating the friction dynamics and ignoring the actuators. Nonlinear simple and complex models of friction in active joints were combined in model-based control schemes in [14], [15] to verify experimentally the significant improvements of path tracking accuracy. Furthermore, a Friedland-Park friction observer as an equivalent friction estimator in joint space was introduced in [16] to produce the friction estimates that help to reject the uncertain frictional disturbance. In [17], a friction model, effective filters and Weighted Least Squares (WLS) identification approach were applied to estimate the dynamic and friction parameters of a parallel manipulator with actuation redundancy controlled by model-based controller resulting in high performances in experimental applications. In [18], the electrical and mechanical dynamics of the actuators were integrated in the dynamic model of a parallel manipulator, and a reduction ratio was considered reflecting the contribution of the joints friction in the inverse dynamics.

In this paper, we propose to investigate both dynamic models of the actuators and the joint frictions in a modelbased control scheme aiming to enhance the overall control performance in terms of precision in high-speed motions. This contribution of the paper was validated through realtime experiments on a non-redundant 4-DoF PKM named VELOCE.

The paper is organized as follows: Section II describes the structure of VELOCE PKM, as well as its kinematic and dynamic modeling. Section III is dedicated to the models of actuator dynamics and joints friction. Section IV shows the design of the proposed control solution. Experimental results are presented and discussed in section V. Section VI concludes the paper and states the future work.

\section{VELOCE PKM: DESCRIPTION AND MODELING}

In the following, a general description of the mechanical structure of VELOCE PKM, shown in Fig. 1, is presented, 
as well as a brief explanation of its kinematic and dynamic models. For more details concerning this work, the reader can refer to [19].

\section{A. Description of VELOCE PKM}

VELOCE robot shown in Fig. 1 is a 4-DOF non-redundant fully actuated parallel manipulator designed at LIRMM (Laboratory of Informatics, Robotics and Microelectronics of Montpellier). It consists of four kinematic chains each is a serial arrangement of an actuator, a rear-arm and a forearm attached to the traveling-plate via spherical joints (see the kinematic diagram of Fig. 2). The moving platform performs three translational motions in the $\mathrm{x}, \mathrm{y}$ and $\mathrm{z}$ axes, and one rotational motion around the $\mathrm{z}$ axis. This moving platform is composed of two parts, upper and lower, assembled in a way such that if they move in a relative motion along a single screw, a rotational motion is generated.

\section{B. Kinematics of VELOCE PKM}

The position and orientation of the moving platform in the three translational and one rotational DoFs is valuated by the 4-dimensional coordinate vector $X=[x, y, z, \alpha]^{T}$. Let $q_{i}$ be the angle of the $i$ th actuator's rotor, then the four joints angles are parametrized by the 4-dimensional coordinate vector $q=\left[q_{1}, q_{2}, q_{3}, q_{4}\right]^{T}$. The proper configuration of the vector $q$ leads to a well positioning of the moving platform in the workspace away from singularities. The geometric study of the structure gives the Forward and Inverse Kinematic relations between $X$ and $q$ respectively. The relation between Cartesian and joints velocities can be expressed as follows:

$$
\dot{q}=J_{m} \dot{X}
$$

where $J_{m} \in \mathfrak{R}^{4 \times 4}$ is the inverse Jacobian Matrix. $\dot{X}$ and $\dot{q}$ are the velocities in Cartesian and joint spaces respectively. It is worth to note that $J_{m}$ is always invertible in the case of non-redundant PKMs that are fed by a reference trajectory away from singularities.

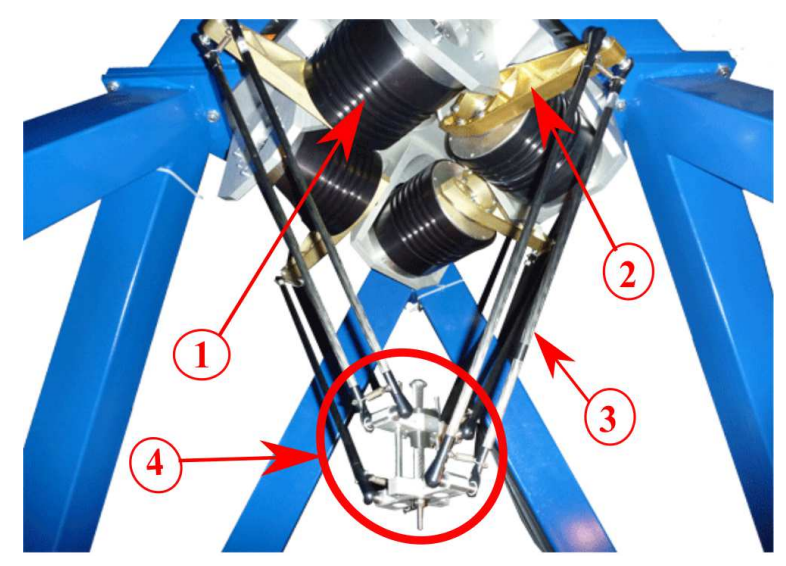

Fig. 1. Side view of VELOCE PKM. 1: Actuator, 2: Rear-arm, 3: Forearm, 4: Moving Platform (called also traveling plate)

\section{Dynamics of VELOCE PKM}

The dynamic model can be obtained by analyzing the dynamics in the joint space and in the traveling-plate space separately, then summing up the two equations of motion. Since VELOCE is a Delta-like parallel robot, the following assumptions proposed in [19] are considered to compute its simplified dynamic model:

Assumption 1: Both dry and viscous frictions in all passive and active joints are neglected.

Assumption 2: The rotational inertia of the forearms is neglected and their mass is split up into two equivalent parts, one part is added to the mass of the arm while the other part is considered with the moving platform.

Regarding the traveling plate, it is subject to three kind of forces: the gravitational forces, the inertial forces and the forces of the payload. Projecting these forces into the joint space using the inverse Jacobian matrix results in the following equations:

$$
\begin{aligned}
\Gamma_{G_{t p}} & =\left(J_{m}^{T}\right)^{-1} M_{t p}[0,0, g, 0]^{T} \\
\Gamma_{F_{t p}} & =\left(J_{m}^{T}\right)^{-1} M_{t p} \ddot{X}
\end{aligned}
$$

where $\Gamma_{G_{t p}}, \Gamma_{F_{t p}} \in \mathfrak{R}^{4}$ are the contributions of the above mentioned forces respectively in each motor torque knowing that $M_{t p} \in \mathfrak{R}^{4 \times 4}$ is the mass matrix of the traveling plate including the mass and inertial effects of the payload. $g=$ $9.81 \mathrm{~m} / \mathrm{s}^{2}$ is the gravity acceleration. From the joints side, the elements that contribute to the motor torques are the inertial forces of the rear-arms and their gravitational forces with the half-masses of the forearms. Those forces can be expressed as follows:

$$
\begin{aligned}
\Gamma_{G_{a}} & =m_{a} r_{a} g \cos (q) \\
\Gamma_{a} & =I_{a} \ddot{q}
\end{aligned}
$$



Fig. 2. Schematic view for one kinematic chain of VElOCE robot. 
where $m_{a}$ is the mass of one rear arm plus the half-mass of one forearm. $r_{a}$ is the distance from the axis of rotation to the center of gravity of the rear arm. $I_{a} \in \mathfrak{R}^{4 \times 4}$ is the diagonal inertia matrix of the rear arm. The inverse dynamic equation can be obtained by summing up the equations from (2) to (5) leading to:

$$
\left(J_{m}^{T}\right)^{-1} M_{t p} \ddot{X}+\Gamma_{G_{t p}}+I_{a} \ddot{q}+\Gamma_{G_{a}}=\Gamma
$$

where $\Gamma \in \mathfrak{R}^{4}$ is the computed torque signal. One can reformulate the inverse dynamic equation using the velocity and acceleration relations between joint and Cartesian spaces, to obtain the standard joint space form of the inverse dynamic model as follows:

$$
M(q) \ddot{q}+C(q, \dot{q}) \dot{q}+G(q)=\Gamma
$$

with $M(q)=I_{a}+\left(J_{m}^{T}\right)^{-1} M_{t p} J_{m}^{-1}$ being the total inertia matrix, $C(q, \dot{q})=-\left(J_{m}^{T}\right)^{-1} M_{t p} J_{m}^{-1} \dot{J}_{m} J_{m}^{-1}$ is the Coriolis and centrifugal forces matrix and $G(q)=\Gamma_{G_{t p}}+\Gamma_{G_{a}}$ be the gravitational forces vector.

\section{Contribution to Dynamic Model}

The mostly used dynamic model in (7) lacks to some factors that adversely affect the power of a controller as the actuator dynamics, articulations friction, transmission system and motor drives. So, our contribution is to model the actuator dynamics and the articulations friction, then synthesis a controller using both models that satisfy our pretend.

\section{A. Actuator Dynamics}

The torque vector in (7) is the torque needed at the level of rotor-rear arm to manipulate the robot as desired, so it is the output torque of the motor. Indeed, the requested torque from the motor $\Gamma_{m}$ by the drive controller is not the same as the output torque, since the internal actuator dynamics appear as a dynamic load in addition to the dynamics of the robot (see Fig. 3). VELOCE robot is driven by direct drive motors (Brushless DC) whose dynamics can be written as [20]:

$$
\Gamma_{m}-\Gamma=J \ddot{q}+B \dot{q}
$$

where $J \in \mathfrak{R}^{4 \times 4}$ is a diagonal matrix representing the total inertia of the actuator (direct-drive motors without gearbox) and the linked load to the rotor (rear arm), and $B \in \mathfrak{R}^{4 \times 4}$ is a diagonal matrix denoted to the damping coefficient in the rotor of the actuator. The rearrangement of (7) and (8) gives us the torque vector that shall be demanded by the drive controller as follows:

$$
\Gamma^{1}=M^{\prime}(q) \ddot{q}+C(q, \dot{q}) \dot{q}+G(q)+B \dot{q}
$$

where $\Gamma^{1}$ is the torque vector including the compensation of actuator dynamics with $M^{\prime}(q)=M(q)+J$ is the rearranged mass and inertia matrix.

\section{B. Friction Dynamics}

According to the mechanical design of VELOCE robot and especially the spherical articulations (passive joints) with self-lubricating, the model proposed in [21] ignoring the friction in passive joints can be used. Then, the friction model contribution can be formulated as follows:

$$
F(\dot{q})=F_{c} \operatorname{sign}(\dot{q})+F_{v} \dot{q}+D
$$

where $F_{c}, F_{v} \in \mathfrak{R}^{4 \times 4}$ are the diagonal matrices of the corresponding coulomb and viscous frictions at the active joints respectively, $D \in \mathfrak{R}^{4}$ is the zero-drift coefficient. Combining this model into (9) and using it in the controller will compensate the aforementioned unfavourable factors.

The coulomb friction is generated from the relative motion of two surfaces (sliding friction). The viscous friction is that between the fluid (air) and the moving boundaries / plates. The zero-drift coefficient is a gradual change in the scale zero of a measuring instrument due to factors such as time, line voltage, or ambient temperature effects. Drift is an indication of the loss of perfect repeatability.

Therefore, we end up with a new dynamic model, including the actuator dynamics and the active joint frictions written as follows:

$$
\Gamma^{2}=M^{\prime}(q) \ddot{q}+C(q, \dot{q}) \dot{q}+G(q)+B \dot{q}+F(\dot{q})
$$

where $\Gamma^{2}$ is the torque vector including the compensation of both actuator and friction dynamics.

\section{Proposed Control SOlution}

A model-based control solution is proposed to validate the positive contribution of the new dynamic model in the control performance. PD controller with Computed Feedforward, a model-based non-adaptive controller constructed from a linear PD controller plus a feedforward term of the dynamics computed along the trajectory $q_{d}$. It is given by [8] in joint space as:

$$
\tau_{P D F F}=\Gamma_{f f}+K_{p} e(t)+K_{v} \dot{e}(t)
$$

such that $\Gamma_{f f}=M\left(q_{d}\right) \ddot{q}_{d}+C\left(q_{d}, \dot{q}_{d}\right) \dot{q}_{d}+G\left(q_{d}\right)$ is the feedforward computed torque from the inverse dynamic model, $e(t)=q_{d}(t)-q(t)$ is the joint tracking position error and $\dot{e}(t)$ is the joint rate error. $K_{p}, K_{v} \in \mathfrak{R}^{4 \times 4}$ are the feedback positive definite matrices. The main advantage of such controller is that the feedforward term is computed from the desired trajectories $q_{d}(t), \dot{q}_{d}(t), \ddot{q}_{d}(t) \in \mathfrak{R}^{4}$ which can be obtained

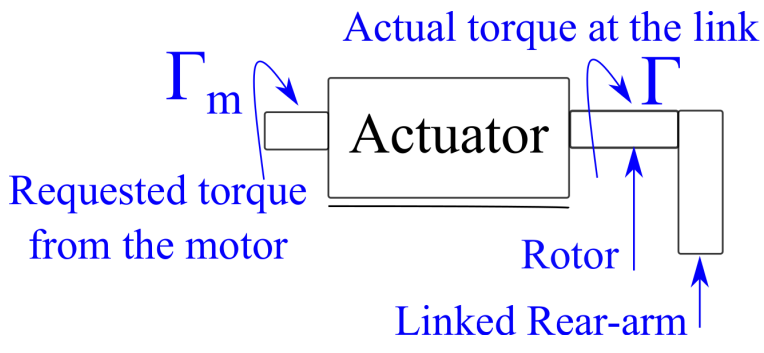

Fig. 3. Mechanical Actuator Dynamics 
off-line and stored in a memory enhancing the execution time.

The contribution to this controller is the use of the new dynamic models formulated in (9) and (11) for the feedforward computation as an extended versions of PDFF control. Then, the generated control inputs will be as following:

$$
\begin{aligned}
& \tau_{E x-P D F F}^{1}=\Gamma_{f f}^{1}+K_{p} e(t)+K_{v} \dot{e}(t) \\
& \tau_{E x-P D F F}^{2}=\Gamma_{f f}^{2}+K_{p} e(t)+K_{v} \dot{e}(t)
\end{aligned}
$$

where $\Gamma_{f f}^{1}, \Gamma_{f f}^{2}$ are the feedforward computed torques from the formulated inverse dynamic models introduced in (9) and (11).

The stability analysis of the PD control with computed feedforward has been reported in [22] showing that the position error will vanish asymptotically in a local sense after selecting properly the feedback matrices.

\section{EXPERIMENTAL RESUlTS}

VELOCE robot is equipped with four TMB0140-1003RBS ETEL direct-drive motors that can provide a peak torque of $127 \mathrm{Nm}$ and reach a maximum speed of 550 rpm. Each motor is supplied with a non-contact incremental optical encoder giving a total number of 5000 pulses per revolution.

To validate the improvement in the performance of PDFF control with the new formulated dynamic models, the standard PDFF and the two extensions of PDFF are implemented on VELOCE robot. The evaluation criteria is considered based on the Root Mean Square of the tracking Error in Translational motion (RMSET) and Rotational motion (RMSER):

$$
\begin{aligned}
& \operatorname{RMSET}=\left(\frac{1}{N} \sum_{i=1}^{N}\left(e_{x}^{2}(i)+e_{y}^{2}(i)+e_{z}^{2}(i)\right)\right)^{1 / 2} \\
& R M S E R=\left(\frac{1}{N} \sum_{i=1}^{N} e_{\alpha}^{2}(i)\right)^{1 / 2}
\end{aligned}
$$

where $N$ is the number of the time-samples, $e_{x}, e_{y}, e_{z}$ denote the tracking error along the $x, y$ and $z$ axes respectively, $e_{\alpha}$ is the tracking error of the platform's rotation, and $i=1, \ldots, 4$

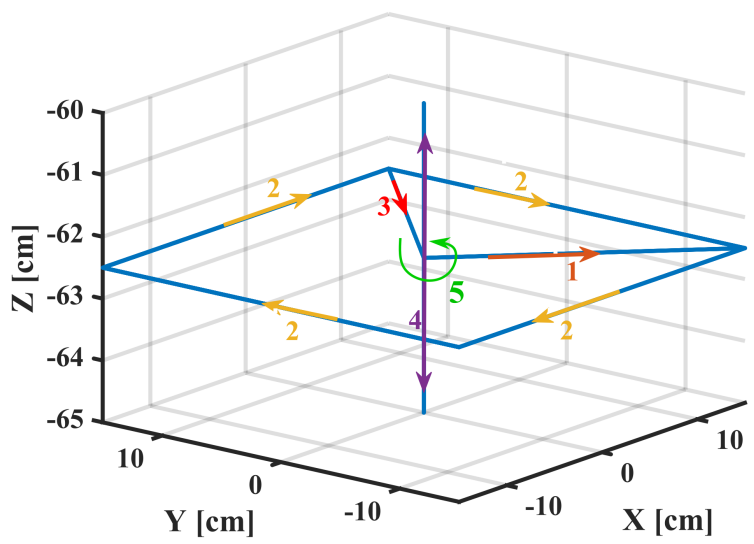

Fig. 4. The desired trajectory in Cartesian space for VELOCE PKM stands for the $i$ th actuator. The feedback gains are evaluated using the Trial-and-Error tuning method which gives $K_{p}=$ 4000 and $K_{v}=6$.

\section{A. Actuator Dynamic Parameters}

The parameters of the actuator dynamics are identified from the provided manuals of the motors driving VELOCE robot. The inertias of the actuator and the rear arm are given as $0.0041 \mathrm{~kg} . \mathrm{m}^{2}$ and $0.0085 \mathrm{~kg} . \mathrm{m}^{2}$ respectively. Then the total inertia calculation gives $J=0.0126 \mathrm{~kg} . \mathrm{m}^{2}$ for each actuator, and the damping coefficient is provided as $B=0.0024$.

\section{B. Friction Parameters Identification}

To identify the parameters of the friction model in (10), a sequence of steps is performed using the experimental testbed and the Matlab/Simulink environment.

1) Apply on VELOCE robot a simple PD controller fed with the desired reference trajectory illustrated in 3D-picture of Fig. 4 in a nominal scenario (used also in the PDFF control experiments).

2) Get out the generated control input $\tau=\Gamma^{2}$ and the measured signal $q$.

3) Estimate from the measured angle $q$ the angular velocity and acceleration $\dot{q}, \ddot{q}$.

4) Compute using the available data this part from (11): $H=M^{\prime}(q) \ddot{q}+C^{\prime}(q, \dot{q}) \dot{q}+G(q)$

5) Write (11) in the following form and substitute the known states:

$$
Y=\Gamma^{2}-H=F_{c} \operatorname{sign}(\dot{q})+F_{v} \dot{q}+D
$$

6) Over $N$ time-samples, (17) can be written as:

$$
\left[\begin{array}{c}
Y_{i, 1} \\
Y_{i, 2} \\
\vdots \\
Y_{i, N}
\end{array}\right]=\left[\begin{array}{ccc}
\operatorname{sign}\left(\dot{q}_{i, 1}\right) & \dot{q}_{i, 1} & 1 \\
\operatorname{sign}\left(\dot{q}_{i, 2}\right) & \dot{q}_{i, 2} & 1 \\
\vdots & \vdots & \vdots \\
\operatorname{sign}\left(\dot{q}_{i, N}\right) & \dot{q}_{i, N} & 1
\end{array}\right]\left[\begin{array}{c}
f_{c_{i}} \\
f_{v_{i}} \\
d_{i}
\end{array}\right]
$$

(18) is displayed in a compact form as: $Y=A X$

7) Apply the Least Square Estimation method to identify $f_{c_{i}}, f_{v_{i}}, d_{i}$ for the $i$ th actuator: $X=\left(A^{T} A\right)^{-1} A^{T} Y$

The results of the identification algorithm of friction parameters are shown in Table I. The large negative deviation in the estimated value of viscous friction may come from the modeling errors or not sufficient exciting trajectories compared with the measurements perturbations.

TABLE I

THE IDENTIFIED FRICTION PARAMETERS OF VELOCE ROBOT.

\begin{tabular}{|cccc|}
\hline Active Joint & $\mathbf{f}_{\mathbf{c}}$ & $\mathbf{f}_{v}$ & $\mathbf{d}$ \\
\hline \hline I & 0.3019 & 0.1198 & 0.1811 \\
II & 0.8879 & 0.2252 & -0.5834 \\
III & 0.0584 & -0.2354 & 0.3194 \\
IV & 0.7104 & 0.3269 & -0.5891 \\
\hline
\end{tabular}



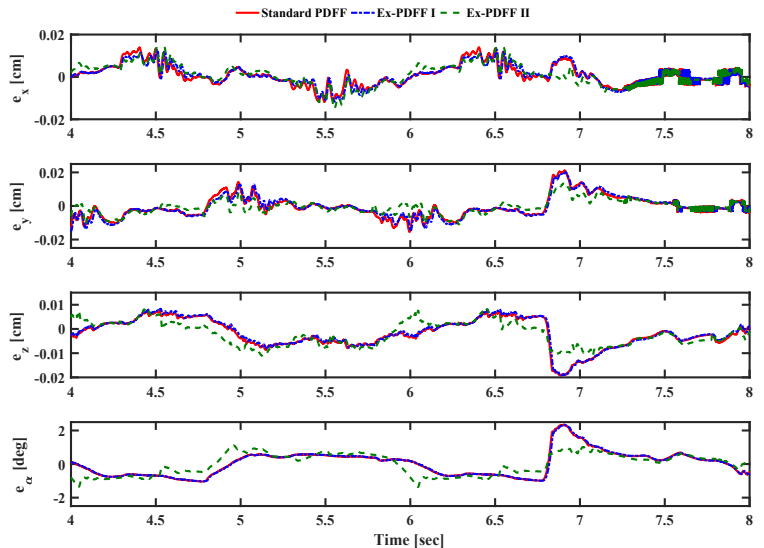

Fig. 5. Scenario 1: Evolution of the Platform's Tracking Error


Fig. 6. Scenario 1: Platform's Tracking Error in a zoomed section

\section{Scenario 1: Nominal Case}

The standard PDFF controller and the two formulated extensions are applied on VELOCE PKM without any payload with a point-to-point motion duration fixed to $T=0.5 \mathrm{~s}$ which gives an acceleration of $1.2 \mathrm{~m} / \mathrm{s}^{2}$. The platform's tracking errors of the controllers are plotted in Fig. 5. For clarification purposes, the plots are zoomed to the interval [4.5,5.5] seconds as shown in Fig. 6. The generated control
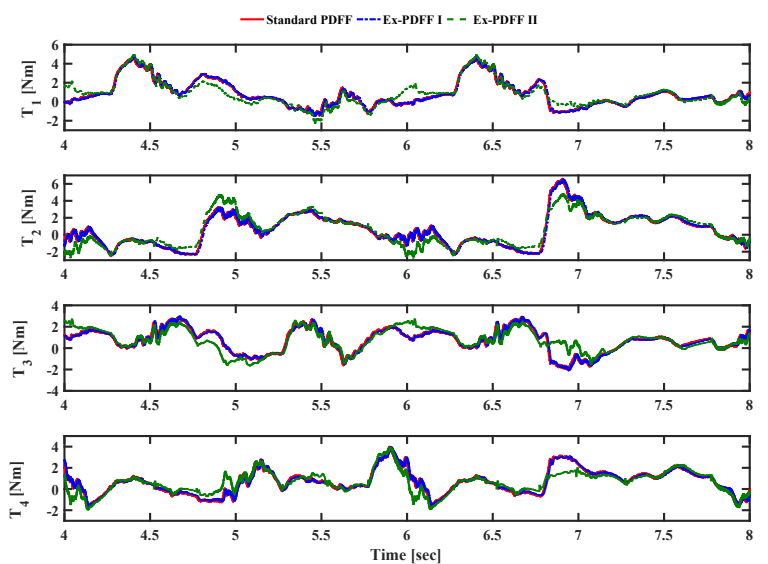

Fig. 7. Scenario 1: Evolution of the Control Inputs
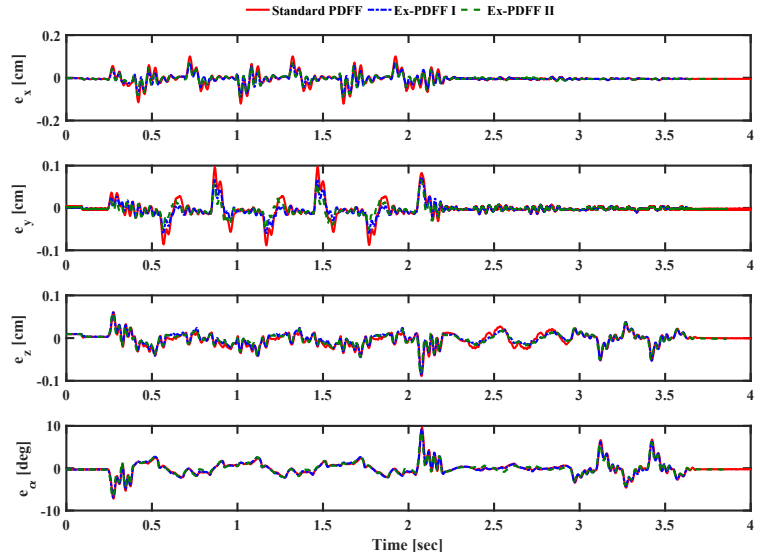

Fig. 8. Scenario 2: Evolution of the Platform's Tracking Error
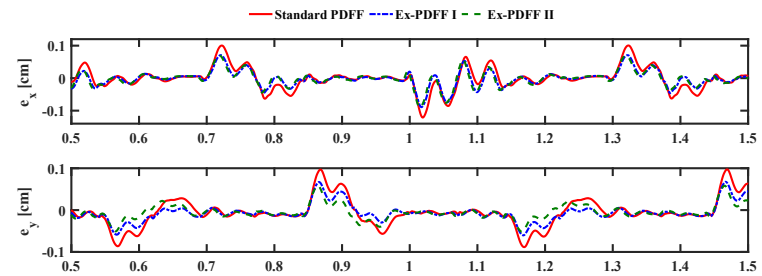



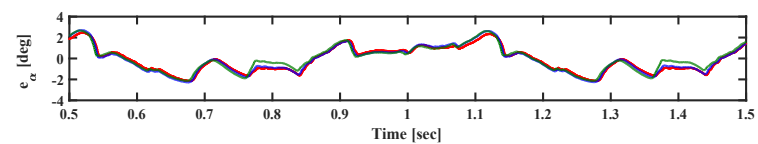

Fig. 9. Scenario 2: Platform's Tracking Error in a zoomed section

input torques of the applied controllers are plotted in Fig. 7, and it is clear that they are in the allowed limited capabilities of the actuators. The root mean squares of the tracking errors show better improvements after considering both actuator and friction dynamics in the closed-loop feedforward control instead of including only the actuator dynamics as shown in Tables II and III.
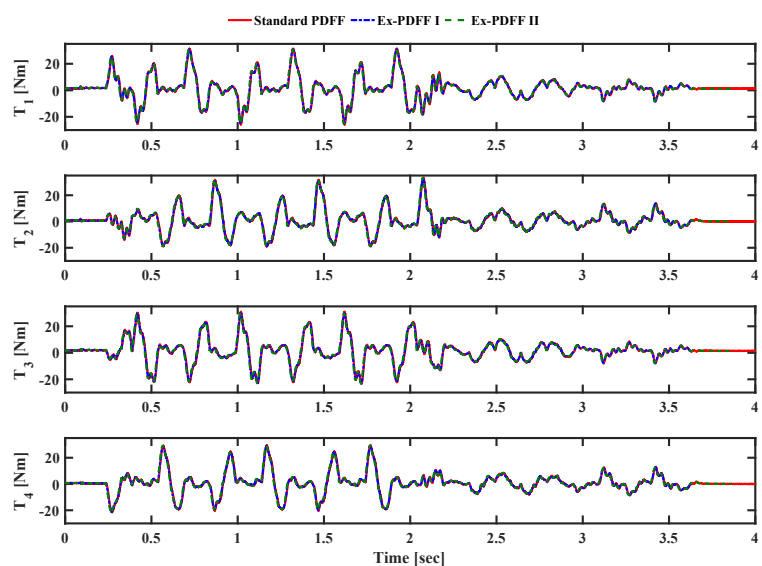

Fig. 10. Scenario 2: Evolution of the Control Inputs 
TABLE II

Control Performance Evaluation of Extended PDFF I

\begin{tabular}{|cccc|}
\hline Scenario & Control & RMSET [cm] & RMSER [deg] \\
\hline \hline \multirow{3}{*}{ Scenario 1 } & Standard PDFF & 0.0089 & 0.7614 \\
& Ex-PDFF I & 0.0084 & 0.7386 \\
& Improvements & $\mathbf{5 . 6 2} \%$ & $\mathbf{3} \%$ \\
\hline \hline \multirow{2}{*}{ Scenario 2 } & Standard PDFF & 0.0349 & 1.5119 \\
& Ex-PDFF I & 0.0251 & 1.4 \\
& Improvements & $\mathbf{2 8 . 0 8} \%$ & $\mathbf{7 . 4} \%$ \\
\hline
\end{tabular}

\section{Scenario 2: Robustness Towards Speed and Payload Variation}

In this scenario, an additional payload of mass $=0.2 \mathrm{Kg}$ is attached to the moving platform of VELOCE robot with an operating speed of $13.3 \mathrm{~m} / \mathrm{s}^{2}(T=0.15 \mathrm{~s})$. The aim of this scenario is to test the robustness against parameters variation especially in pick-and-place applications which need high response and swiftness. The platform's tracking errors are exposed in Fig. 8 with a zoomed interval $[0.5,1.5]$ seconds shown in Fig. 9. The generated control input torques are also under the saturation limits of the motors as shown in Fig. 10. The results show that the contribution of both actuator and friction dynamics promotes the performance of the PDFF controller up to $33.81 \%$ and $17.56 \%$ for translational and rotational motions respectively (see Table III). While the actuator dynamics enclosed in the control-loop alone without friction rises the performance up to $28.08 \%$ for translational motions and $7.4 \%$ for rotational(see Table II).

We can say that a model-based controller relies on a comprehensive dynamic model including both of actuator and friction dynamics can enhance the performance of parallel robots in terms of precision, speed variation and robustness.

\section{CONCLUSiOnS AND Future WORK}

In this paper, a new formulation of the dynamic model of PKMs have been proposed including the friction and actuator dynamics. The contribution was to compensate the effects of the aforementioned dynamics in addition to the high nonlinearities exist in PKMs. A PD with computed feedforward model-based controller have been applied enclosing the formulated dynamics. The results validated that modelbased controllers rely on more comprehensive dynamics outperform the simplified ones in terms of tracking performance when operating in real-time conditions. Moreover, the robustness of the proposed control solution towards payload and speed variation has been proved. For future work, one can use the proposed dynamic model in adaptive control algorithms performing an online estimation for the friction parameters. Adaptive model-based controllers compensate for the time-varying parameters and uncertainties of PKMs attaining better control performance and more robustness.

\section{ACKNOWLEDGMENT}

This work is supported by the ARPE-ARROW project.

\section{REFERENCES}

[1] J.-P. Merlet, Parallel Robots, 2nd ed., ser. Solid Mechanics And Its Applications, G. GLADWELL, Ed. The NetherLands: Springer, 2006, vol. 128.
TABLE III

Control PERformance Evaluation of EXtended PDFF II

\begin{tabular}{|cccc|}
\hline Scenario & Control & RMSET [cm] & RMSER [deg] \\
\hline \hline \multirow{3}{*}{ Scenario 1 } & Standard PDFF & 0.0089 & 0.7614 \\
& Ex-PDFF II & 0.0072 & 0.5661 \\
& Improvements & $\mathbf{1 9 . 1} \%$ & $\mathbf{2 5 . 6 5} \%$ \\
\hline \hline \multirow{2}{*}{ Scenario 2 } & Standard PDFF & 0.0349 & 1.5119 \\
& Ex-PDFF II & 0.0231 & 1.2464 \\
& Improvements & $\mathbf{3 3 . 8 1} \%$ & $\mathbf{1 7 . 5 6} \%$ \\
\hline
\end{tabular}

[2] S. Kucuk, Ed., Serial and Parallel Robot Manipulators - Kinematics, Dynamics, Control and Optimization. Croatia: InTech, 2012.

[3] D. Stewart, "A platform with six degrees of freedom," ARCHIVE: Proceedings of the Institution of Mechanical Engineers 1847-1982, vol. 180, pp. 371-386, June 1965.

[4] T. Toyama, Y. Yamakawa, and H. Suzuki, "Machine tool having parallel structure," Patent 5715729 , Feb. 10, 1998.

[5] L. Yangmin and X. Qingsong, "Design and development of a medical parallel robot for cardiopulmonary resuscitation," IEEE/ASME Transactions on Mechatronics, vol. 12, pp. 265-273, June 2007.

[6] G. S. Natal, A. Chemori, and F. Pierrot, "Nonlinear control of parallel manipulators for very high accelerations without velocity measurement: Stability analysis and experiments on par2 parallel manipulator,' Robotica, vol. 34, pp. 43-70, Jan 2016.

[7] M. Bennehar, A. Chemori, and F. Pierrot, "A new revised desired compensation adaptive control for enhanced tracking: application to ra-pkms," Advanced Robotics, vol. 30, pp. 1199-1214, June 2016.

[8] G. S. Natal, A. Chemori, and F. Pierrot, "Dual-space control of extremely fast parallel manipulators: Payload changes and the $100 \mathrm{~g}$ experiment," IEEE Transactions on Control Systems Technology, vol. 23, pp. 1520-1535, July 2015

[9] M. Bennehar, A. Chemori, M. Bouri, L. Jenni, and F. Pierrot, "A new rise-based adaptive control of pkms: design, stability analysis and experiments," International Journal of Control, vol. 91, pp. 593-607, Feb 2017.

[10] H. Saied, A. Chemori, M. E. Rafei, C. Francis, and F. Pierrot, "From non-model-based to model-based control of pkms: A comparative study," in ICAMMRMS: International Congress for the Advancement of Mechanism, Machine, Robotics and Mechatronics Sciences, Beirut, Lebanon, Oct 2017, pp. 59-64.

[11] C. Su and Y. Stepanenko, "Backstepping based hybrid adaptive control of robot manipulators incorporating actuator dynamics," in Proceedings of the 1996 IEEE IECON 22nd International Conference on Industrial Electronics, Control, and Instrumentation, Taipei, Taiwan, Aug 1996, pp. 141-153.

[12] A. Lotfazar, M. Eghtesad, and M. Mohseni, "Integrator backstepping control of a 5 dof robot manipulator incorporating actuator dynamics," in Proceedings of 2003 IEEE Conference on Control Applications, Istanbul, Turkey, June 2003, pp. 1007-1012.

[13] C. An, C. Atkeson, J. Griffiths, and J. Hollerbach, "Experimental evaluation of feedforward and computed torque control," IEEE Transactions on Robotics and Automation, vol. 5, pp. 368-373, Jun 2008.

[14] M. Grotjahn, B. Heimann, and H. Abdellatif, "Identification of friction and rigid-body dynamics of parallel kinematic structures for modelbased control,' Multibody System Dynamics, vol. 11, pp. 273-294, April 2004.

[15] W. Shang, S. Cong, and Y. Zhang, "Nonlinear friction compensation of a 2-dof planar parallel manipulator," Mechatronics, vol. 18-7, pp. 340-346, Sep 2008.

[16] H. Kim, Y. Cho, and K.-I. Lee, "Robust nonlinear task space control for 6 dof parallel manipulator," Automatica, vol. 41, pp. 1591-1600, Sep 2005.

[17] W. Shang, S. Cong, and Y. Zhang, "Identification of dynamic and friction parameters of a parallel manipulator with actuation redundancy," Mechatronics, vol. 20-2, pp. 192-200, March 2010.

[18] Z. Yang, J. Wu, J. Mei, J. Gao, and T. Huang, "Mechatronic model based computed torque control of a parallel manipulator," International journal of advanced robotic systems, vol. 5, pp. 123-128, Mar 1989.

[19] M. Bennehar, "Some contributions to nonlinear adaptive control of pkms : From design to real-time experiments," Ph.D. dissertation, University of Montpellier 2, France, December 2015. [Online]. Available: https://tel.archives-ouvertes.fr/tel-01346432/

[20] Z. Shao, "Identification and control of low-cost robot manipulators," Ph.D. dissertation, Ecole Centrale de Lille, France, March 2016. [Online]. Available: https://tel.archives-ouvertes.fr/tel-01484288/

[21] W. Shang and S. Cong, "Serial and parallel robot manipulators kinematics, dynamics, control and optimization," in Nonlinear Dynamic Control and Friction Compensation of Parallel Manipulators S. Kucuk, Ed. Croatia: InTech, 2012, ch. 12, pp. 231-252.

[22] F. Reyes and R. Kelly, "Experimental evaluation of model-based controllers on a direct-drive robot arm," Mechatronics, vol. 11, pp. 267-282, April 2001. 